\title{
THE ANATOMY AND PHYSICS OF THE TEMPOROMANDIBULAR JOINT.
}

\author{
By George H. Wilson, D. D.S., Cleveland, Ohio.
}

(Read before the National Dental Association at Its Twenty-third Annual Session, New Orleans, La., October 20-24, 1919.)

I T MAY be stated as a truism that the anatomy and physiology of the temporomandibular joint are constants, that thruout a period of many generations there is no appreciable change. However, it must always be recognized that there are many variations from the fundamental type; but, that the underlying principle is the same in all cases. It has been said that variation in anatomy is the rule, and the type the exception. Accepting the statement as in a broad sense true, the motive of this paper will be to determine the underlying principles of the function of mastication.

It is recognized also that sciences are developed by stages, that the first statement of a truth is hedged about by errors which are gradually eliminated. For instance, the arteries were considered as air passages, hence the name; the physiological differences of the human organism were attributed to the "humors" of the body, hence the temperaments; and the mandible was considered as a lever of the third class.

The subject of this paper in its most concrete form is: THE MANDIBLE IS NOT A LEVER OF THE THIRD CLASS.

It is the purpose of this paper to detail the evidence upon which this statement is based and thereby establish its soundness; also to direct attention to its far reaching importance in prosthetic dentistry.

Chart I, will fix in the mind the three classes of levers.

Class one need not be considered, classes two and three are of some importance in this study. They are incidentally but not primarily factors. In class three the power is between the fulcrum and the work, thus the power is expended between the fulcrum and the work. If the function of the superficial portion of the masseter and internal pterygoid only were involved, then the mandible would be a lever of the third class, and from one-fourth to threefourths of the power would be expended upon the fulcrum (the condyle). This would imply a great sacrifice of energy, and a destruction of tissue. The sacrifice of energy is self evident, and the destruction of tissue has been demonstrated by Prof. H. J. Prentiss, of the University of Iowa, and will be referred to later.

For a clear mental vision of the temporomandibular joint, a resume of the anatomy of the parts is presented.*

\section{CHART II.}

The joint consists of the Mandibular Fossa of the temporal bone, the Condyle of the Mandible, the Articular Disk, and

*The nomenclature and quotations of descriptive anatomy are taken from Gray, Twentieth Edition, 1918, revised and re-edited by Prof. Lewis, of Johns Hopkins University. 
the two synovial membranes, the upper and lower. These parts are all enclosed in the Articular Capsule.

The auxiliary ligaments are: Temporomandibular, Sphenomandibular and Stylomandibular.

The chief muscles of mastication are: Masseter, Temporalis, Pterygoideus externus, and Pterygoideus internus.

The auxiliary muscles of mastication to the lower of the two joints. On the other hand, when the mouth is opened more widely, both joints are concerned in the movement; in the lower joint the movement is of a hinge-like character, the condyle moves around a transverse axis on the disk, while in the upper joint, the movement is of a gliding character, the disk together with the condyle, gliding forward on the articular

CHART 1 ,
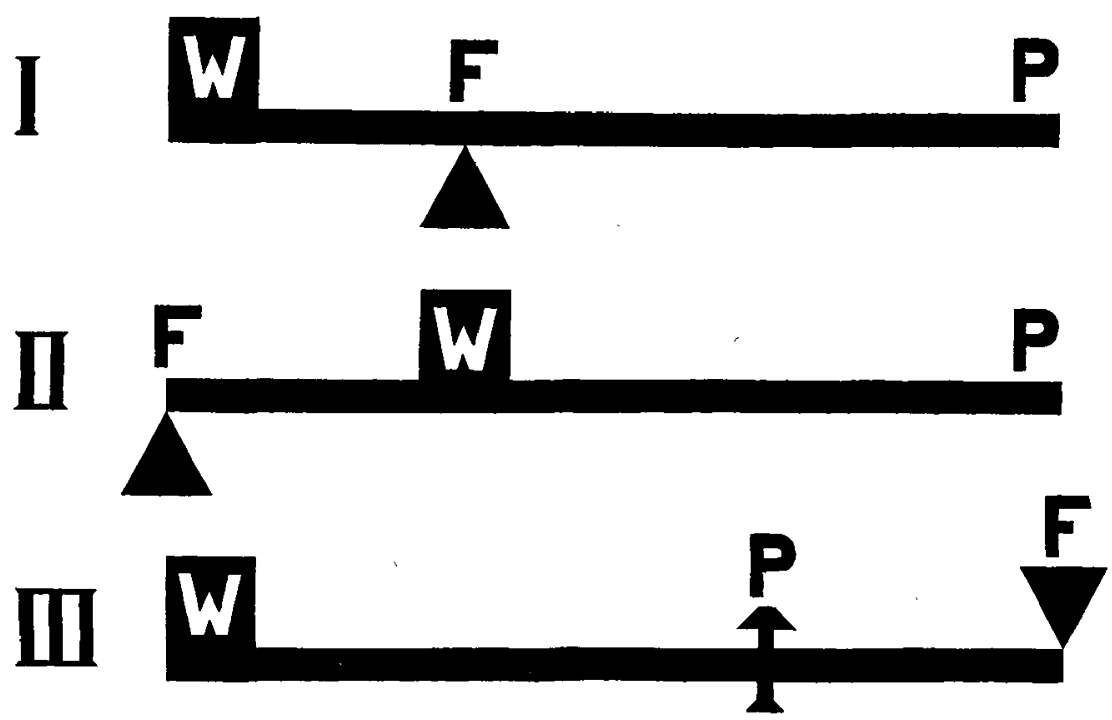

are: Platisma, Digastricus, Mylohyideus, and Geniohoideus.

Gray says (Page 298): "The movements permitted in this articulation are extensive. Thus, the mandible may be depressed or elevated, or carried forward or backward; a slight amount of sideto-side movement is also permitted. It must be borne in mind that there are two distinct joints in this articulationone between the condyle and the articular disk, and another between the disk and the mandibular fossa. When the mouth is slightly opened, as during ordinary conversation, the movement is confined tubercle, around an axis which passes thru the mandibular foramina. These two movements take place simultaneously, the condyle and disk moving forward on the eminence, and at the same time the condyle revolves on the disk. In shutting the mouth, the reverse action takes place; the disk glides back, carrying the condyle with it, and this at the same time moves back to its former position. When the mandible is carried horizontally forward, as in protruding the lower incisor teeth in front of the upper, the movement takes place principally in the upper joint, the disk and 
condyle gliding forward in the mandibular fossa on the articular tubercle. The grinding or chewing movement is produced by one condyle, with its disk, gliding alternately forward and backward, while the other condyle moves drawn forward by the simultaneous action of the Pterygoideus internus and externus, the superficial fibres of the Masseter and the anterior fibers of the Temporalis; and backward by the deep fibers of the Masseter and the posterior

CHART 2.

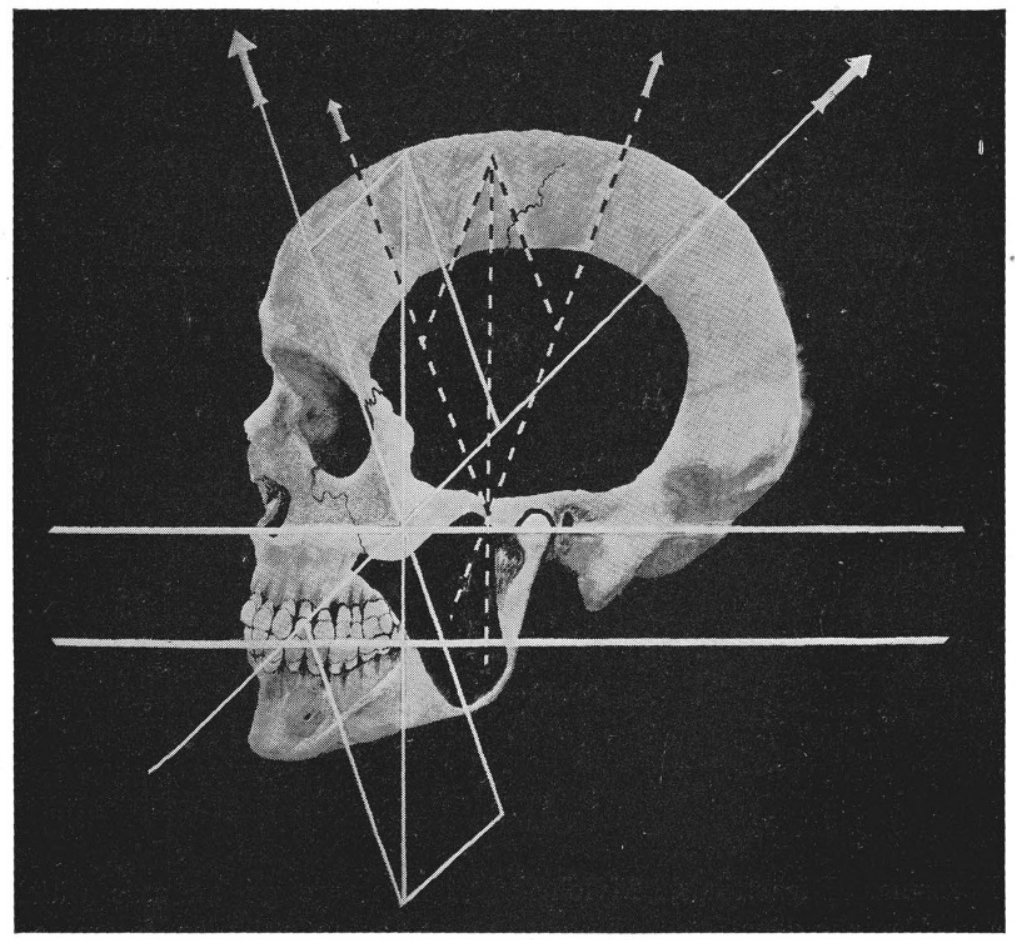

simultaneously in the opposite direction; at the same time the condyle undergoes a vertical rotation on the disk. One condyle advances and rotates, the other condyle recedes and rotates, in alternate succession.

"The mandible is depressed by its own weight, assisted by the Platisma, the Digastricus, the Mylohyoideus, and the Geniohyoideus. It is elevated by the Masseter, Pterygoideus internus, and the anterior part of Temporalis. It is fibres of the Temporalis. The grinding movement is caused by the alternate action of the Pterygoidei of either side."

This statement is concise and probably is the latest accepted description by anatomists of the composition and action of the Temporomandibular joint. The study of the physics of the individual muscles and of their combined action, will help to develop an understanding of the function of the masticatory apparatus. 
The function of a muscle fiber is to draw its inserted end towards its origin in a straight line. Two or more muscles that are not parallel but acting simultaneously will produce a resultant action. The resultant action is influenced by the ratio of the power of the muscles involved as well as by the direction of their individual activities. It is by the result of the combined action of the muscles that their function is determined. Therefore, the parallelogram of forces will demonstrate the physics of mastication and the principle included.

The superficial portion of the masseter, and the internal pterygoid muscles act in conjunction with each other in a direct thrust, and in a vertical and forward direction, at approximately an angle of twenty-five (25) degrees from the perpendicular. (See Chart 2.) The posterior portion of the temporal muscle acts also in the direct closing thrust of the mandible; however, its line of force is upward, and about forty-five (45) degrees from the perpendicular backward. (See Chart 2). As the power of the combined action of the masseter and the internal pterygoid is two or three times as great as that of the temporal muscle, the parallel lines of the parallelogram representing the thrust of these muscles must be two to three times as long as the parallel lines representing the thrust of the temporal muscle; therefore, the diagonal line of an oblique parallelogram will represent the resultant of the action of these three muscles. As the resultant is at right angles to the occlusal plane, it is evident that the whole force of these muscles is expended upon the bolus of food and not any portion of it upon the condyle. Therefore, the condyle is not a fulcrum and the mandible is not a lever of the third class.

This diagram is the true physical demonstration of the action of the muscles, however, a variation of the demonstration may be more easily comprehended. As force acts in a straight line, and a force acting upon a solid body acts upon every portion of that solid body in parallel lines; therefore the action of the temporal muscle attached to the coronoid process is just the same as if the attachment were carried forward to the apices of the incisor teeth. By constructing the parallelogram below, and to this extended line, it may be easier to conceive of the resultant force being at right angles to the occlusal plane. (See Chart).

A further proof that the mandible is not a lever of the third class is found in the parallelogram of forces constructed upon the lines of force of the deep portion of the masseter which draws the mandible upward and backward; and the anterior portion of the temporal muscle which draws the mandible upward and forward. The resultant force of these minor portions is in the same direction as the resultant force of the major portions of these muscles; however, they act as a stabilizer, and make more positive the intent of the action of these muscles. (See broken lines of Chart 2.)

Another positive evidence that the mandible is not a lever of the third class is found in the action of the external pterygoid muscle. This muscle is inserted into the neck of the condyloid process of the mandible and into the articular disk. (See Figure a, Chart 3.)

\section{Chart III.}

The function of this muscle is to draw the mandible and articular disk forward and inward. This results in drawing the condyle and articular disk, with its lubricating sacks, on to the articular tubercle (eminentia articularis). Therefore, if the mandible is a lever of the 
third class, then the fulcrum consists of a lubricated inclined plane, a mechanical construction unthinkable of our conception of the Omnipotent. Architect. Hence, if the articular tubercle does not function as a fulcrum, the mandible does not function as a lever of the third class.

Another very strong evidence that the mandible is not primarily a lever of the third class was presented by H. J. Prentiss, M. D., Professor of Anatomy,
This is positive evidence that the action of the mandible is not primarily a lever of the third class, and that when it does become such, as the sequence of the loss of the teeth, then it results in a greater, or less destruction of the articular disk.

These four evidences show conclusively that nature did not design the mandible as a lever, but as the moving part of a mill.

Consider the masticatory apparatus of

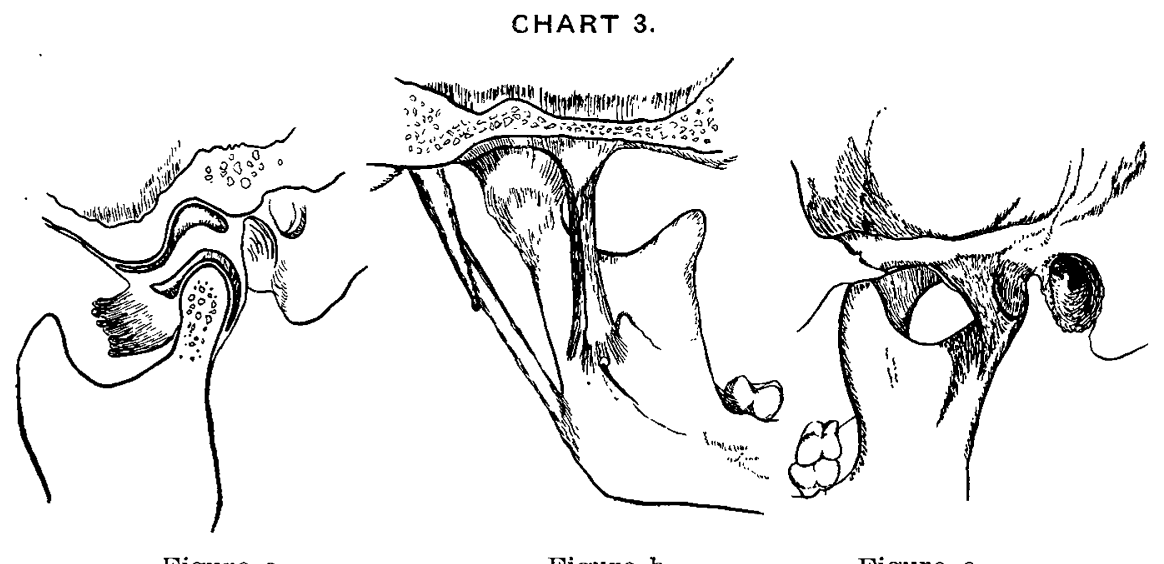

Figure a.

Figure b.

Figure c.

at the University of Iowa, in a paper published in the Dental Cosmos, June 1918. This is an exceedingly interesting article and can be read with profit by every member of the dental profession. The paper is based entirely upon laboratory investigations of various joints. He considers the histology of the knee joint from early embryonic to adult life. He says: "We see therefore in the knee joint a retrograde absorption of the meniscus (articular disk) which is necessary to a normal function of the joint. The sequence of events just followed in this joint (knee) may be traced to the mandibular joint, only here it becomes pathological with the loss of the teeth." man as a mill. This can best be done by inverting the head, when the maxilla become the nether millstone and the mandible the upper and movable millstone; the maxillary molars and bicuspids represent the convex surface, and the molars and bicuspids of the mandible represent the concave rotating or triturating surface; the rami are balancing arms supported by ball and socket joints, the movements of these joints are limited in scope by the ligaments; and the milling power is furnished by the muscles.

This arrangement would result in the loss of gravitation and require more power in separating the millstones for the insertion of the grist, and would place 
more pressure upon the articular disk causing the perforation of the articular disk to become a physiological process, which is not the intent of nature. Nature reversed this order and suspended the movable millstone, thus conserving energy, reducing friction and preserving tissue. This suggests the function of the ligaments and facia. This principle of suspension in mechanics is used in some of the greatest engineering schemes of man. For instance, the electric power plant at Niagara Falls, where the generating power consists of a column of water falling 185 feet and entering the turbine wheel from beneath, thereby reducing friction to a minimum and providing a maximum of energy.

The last of the assemblage of factors to be presented as proof that the mandible is not primarily a lever of the third class is the purpose and action of the ligaments. 'The purpose of ligaments is to act in the capacity of a rope. They are flexible, but neither elastic nor contractile; they act to suspend and to restrict. The action of the temporomandibular ligament should be elucidated. The quotation from Gray's Anatomy, previously given, states: "In the upper joint, the movement is of a gliding character, the disk, together with the condyle, gliding forward on to the articular tubercle, around an axis which passed thru the mandibular foramina." The location of this axis is an error, which is based upon the supposition that the stylomandibular ligament is the fixation forming this axis. (Figure b, Chart 3.)

Such an action is an impossibility, for if this rope-like attachment existed the mandible could not be protruded. It is the temporomandibular (external oblique) ligament that performs the function of the axis of rotation of the upper joint. In opening the mouth, the head of the condyle first rotates on an axis on the under surface of the articular disk, as stated by Gray, when the temporomandibular ligament is made taut, its insertion at the neck of the condyle becomes the axis of rotation, and the head of the condyle and articular disk travel forward on to the articular eminence. (Figure c, Chart 3.)

As this ligament passes from the zygomatic process of the temporal bone downward and backward to the neck of the condyle its pendulous nature permits protrusion of the mandible, whereas the fibres of the stylo-mandibular ligament pass downward and forward and cannot act as the axis of rotation and permit protrusion. Therefore, as it permits protrusion it is not the axis of rotation.

The stylomandibular and sphenomandibular ligaments are long thickened bands of Fascia Colli (deep cervical fascia) and function as pendulous suspensors. Incidentally these ligaments, with the other temporomandibular ligaments, may aid the mandible in acting as a lever of the second class, just as certain conditions may arise so as to cause the mandible to act as a lever of the third class; but these are not the primary or normal functions. Therefore, the temporomandibular joint is a compensating joint, and designed to aid the various factors in the masticatory apparatus in conforming to requirements.

This evidence and reasoning should and does establish the soundness of the statement that the "Mandible is not a lever of the third class."

The first purpose of this paper being accomplished, the second can be disposed of quickly.

It is an axiom of logic that-If the premise of a proposition is false, the 
conclusion is false. Therefore, as the teaching of, and the instruments for the anatomical articulation of artificial teeth are based upon a false proposition, it follows that the methods of construction of artificial dentures are wrong. However, the profession is deeply indebted to the late Dr. Bonwill, for his pioneer work; Prof. Gysi for his investigations and especially for his demonstration of the variable rotating centers; $\mathrm{Mr}$. Norman G. Bennett for his demonstration of the lateral mandibular movements; and Dr. Rupert E. Hall for his practical application of the correct principles, and his demonstration of the falseness of some of the supposed principles of denture construction.

It is a logical conclusion that the movements of the temporo-mandibular joint are of very minor importance, valuable only as a confirmation of the action of the muscles and the restrain- ing influence of the bone surfaces and ligaments. The sequence is that the millstone (the teeth), their position and surfaces, their stability, and the direction of the power employed are the essential factors in the mechanical construction of artificial dentures. It is always apropos to mention the esthetics of artificial dentures, therefore the essential factors in denture restorations are a thoro comprehinsion of the anatomy, physics and esthetics; and a suitable technic.

If this paper has established a sound, sane and scientific premise for the future teaching of dental prosthetics, its purpose has been accomplished.

P. S.-As this paper goes to press, the development and function of the temporomandibular ligaments are being questioned and subjected to further study. Whether the ligament exists, or not, makes no practical difference to the prosthetists.-Author. 